[2] Y. Kameda, N. Asano, M. Yoshikawa, K. Matsui, J. Antibiot. 33 (1980) 1575: T. Takehara, E. Newbrun, C. I. Hoover, Caries Res. 19 (1985) 266

[3] T. Isawa, H. Yamamoto, M. Shibata, J. Antibiot. 23 (1970) 595

[4] A. Köhn, R. R. Schmidt, Liebigs Ann. Chem. 1985, 775, zit. Lit.

[5] H. Paulsen, F. R. Heiker, Angew. Chem. 92 (1980) 930; Angew. Chem. Int. Ed. Engl. 19 (1980) 904; Liebigs Ann. Chem. 1981. 2180.

[6] S. Ogawa, T. Toyokuni, T. Suami, Chem. Lett. 1980, 713; 1981, 947; S Ogawa, T. Toyokuni, M. Omata, N. Chida, T. Suami, Bull. Chem. Soc. Jpn. 53 (1980) 455; S. Ogawa, N. Chida, T. Suami, J. Org. Chem. 48 (1983) 1203: T. Toyokuni, S. Ogawa, T. Suami, Bull. Chem. Soc. Jpn. 56 (1983) 1161.

[7] N. Sakairi, H. Kuzuhara, Tetrahedron Lett. 23 (1982) 5327.

[8] W. Ando, Acc. Chem. Res. 10 (1977) 179; R. W. Hoffmann, Angew. Chem. 91 (1979) 625; Angew. Chem. Int. Ed. Engl. 18 (1979) 563.

[9] A. Köhn, Dissertation, Universităt Konstanz 1987

[10] D. Semeira, M. Phillippe, J.-M. Delaumeny, A.-M. Sepulchre, D. Gero, Synthesis 1983, 710. Diese Methode wurde verbessert: A. Köhn, unveröfentlichte Ergebnisse (1984) (siehe [9]); S. Mirza, L.-P. Molleyres, A Vasella, Helv. Chim. Acta 68 (1985) 989.

[1I] M. T. Reetz, H. Müller-Starke, Tetrahedron Lett. 25 (1984) 330 I.

[12] E. J. Reist, V. J. Bartuska, L. Goodman, J. Org. Chem. 29 (1964) 3725: V. G. Nayah, R. C. Whistler, ibid. 34 (1969) 97.

\section{Enzymkatalysierte Cyanhydrin-Synthese in organischen Lösungsmitteln}

\section{Von Franz Effenberger*, Thomas Ziegler und Siegfried Förster}

Pfeil et al. haben die enantioselektive Addition von Blausäure 2 an Benzaldehyd und zahlreiche andere Aldehyde 1 in Gegenwart des Enzyms Mandelonitril-Lyase $(,(R)$-Oxynitrilase“") zu optisch aktiven $(R)$-Cyanhydrinen 3 beschrieben ${ }^{[1]}$. In den bisher ausschließlich angewendeten wäßrigen oder wäßrig-alkoholischen Systemen läuft jedoch neben der enzymkatalysierten Addition auch die chemische Addition ab, die zu Racematen führt, so daß häufig nur mäßige optische Ausbeuten bei der Reaktion erhalten werden ${ }^{|l c|}$<smiles>[R]C=[C+]C#N</smiles>

Die leichte Zugänglichkeit ${ }^{[2]}$ der Mandelonitril-Lyase (E.C. 4.1.2.10) aus Bittermandeln (prunus amygdalus) und die große Bedeutung optisch aktiver Cyanhydrine zur Gewinnung optisch aktiver Aminoalkohole ${ }^{[l c]}, \alpha$-Hydroxycarbonsäuren, Pyrethroid-Insektizide ${ }^{[3 a]}$, Imidazole und Heterocyclen ${ }^{(3 b)}$ veranlaßte uns, diese Reaktion eingehender zu untersuchen. Unser Ziel war die präparative Herstellung von Cyanhydrinen mit möglichst hoher Enantiomerenreinheit.

In Wasser/Ethanol führte die Variation der Reaktionsbedingungen ( $\mathrm{pH}$-Wert, Temperatur, Konzentration) zu keinen wesentlichen Verbesserungen gegenüber den früheren Befunden ${ }^{[1]}$. Bei Verwendung von organischen, nicht mit Wasser mischbaren Lösungsmitteln, in denen enzymkatalysierte Reaktionen ebenfalls ablaufen können ${ }^{[4]}$, wird die chemische Reaktion jedoch weitgehend unterdrückt, während die enzymatische Cyanhydrinbildung nur etwas verlangsamt wird (Abb. 1). Dadurch nimmt die Enantiomerenreinheit der Cyanhydrine deutlich zu.

[*] Prof. Dr. F. Effenberger, Dr. T. Ziegler, Dr. S. Förster Institut für Organische Chemie der Universität Pfaffenwaldring 55, D-7000 Stuttgart 80
Unter den von uns geprüften organischen Lösungsmitteln erwies sich Ethylacetat als am besten geeignet; die erzielte Enantiomerenreinheit ist höher als in $\mathrm{H}_{2} \mathrm{O} / \mathrm{EtOH}$. Das Enzym kann dabei auf Trägern fixiert eingesetzt werden, es ist reisolierbar und kann wiederverwendet werden. Von den untersuchten Trägermaterialien ECTEOLA-Cellulose, DEAE-Cellulose, Glasperlen und Cellulose eignet sich Cellulose am besten.

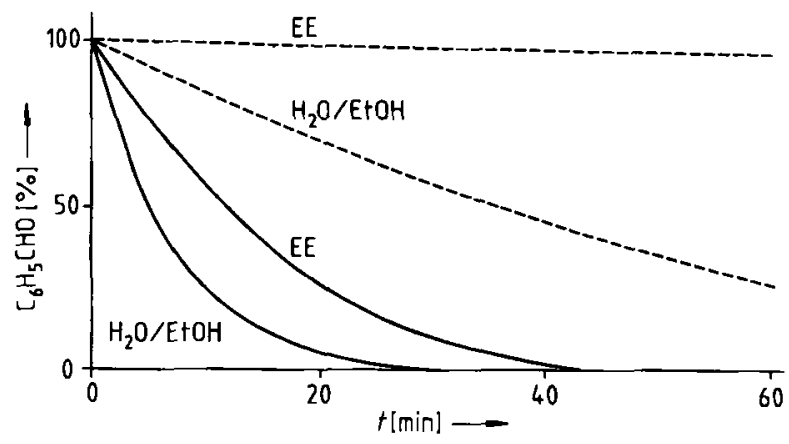

Abb. 1. Geschwindigkeit der chemischen (---) und enzymatischen Addition (-) von $\mathrm{HCN}$ an Benzaldehyd (Anfangskonzentration $5 \cdot 10^{-3} \mathrm{M}$ ) in $\mathrm{H}_{2} \mathrm{O}$ / EtOH und in Ethylacetat(EE)/Celiulose.

Bei den früheren Untersuchungen ${ }^{[1]}$ war lediglich aus den Drehwerten der erhaltenen Produkte auf die optische Ausbeute geschlossen worden. Da nur Mandelsăurenitril in reiner Form bekannt war, war nur im Falle der Reaktion von Benzaldehyd mit Blausäure auf diese Weise eine Aussage über die optische Reinheit des Produkts möglich; im allgemeinen lassen aber die Drehwerte keine genauen Aussagen über die optische Reinheit einer Verbindung zu. AuBerdem können Cyanhydrine sehr leicht über die Gleichgewichtsreaktion racemisieren. Wir haben daher die erhaltenen Cyanhydrine mit $(R)-\alpha$-Methoxy- $\alpha$-trifluormethylphenylessigsăurechlorid $[R(+) \text {-MTPA-Chlorid }]^{[\zeta]}$ in die diastereomeren Ester überführt und deren optische Reinheit gaschromatographisch ermittelt. In Tabelle 1 sind die Ergebnisse der von uns durchgeführten enzymatischen Cyanhydrinsynthesen einerseits in $\mathrm{H}_{2} \mathrm{O} / \mathrm{EtOH}$ (nach ${ }^{\text {III) }}$ und andererseits in Essigester einander gegenübergestellt.

Tabelle 1. Enzymatische Bildung von Cyanhydrinen 3 in $\mathrm{H}_{2} \mathrm{O} / \mathrm{EtOH}$ (siehe Arbeitsvorschrift $\mathrm{A}$ ) und in Ethylacetat(EE)/Cellulose (siehe Arbeitsvorschrift B).

\begin{tabular}{|c|c|c|c|c|c|c|}
\hline \multirow[b]{2}{*}{ Aldehyd 1} & \multicolumn{3}{|c|}{3 in $\mathrm{H}_{2} \mathrm{O} / \mathrm{EtOH}$} & \multicolumn{3}{|c|}{3 in EE/Cellulose } \\
\hline & $\begin{array}{l}\text { Reakt.- } \\
\text { zeit } \\
\text { [h] }\end{array}$ & $\begin{array}{l}\text { Ausb. } \\
{[\%]}\end{array}$ & $\begin{array}{l}e e[\mathrm{a}] \\
{[\%]}\end{array}$ & $\begin{array}{l}\text { Reakt.- } \\
\text { zeit } \\
\text { [h] }\end{array}$ & $\begin{array}{l}\text { Ausb. } \\
\text { [\%] }\end{array}$ & $\begin{array}{l}e e[\mathrm{a}] \\
{[\%]}\end{array}$ \\
\hline Benzaldehyd & 1 & 99 & 86 & 2.5 & 95 & 99 \\
\hline $\begin{array}{l}\text { 3-Phenoxy- } \\
\text { benzaldehyd }\end{array}$ & 5 & 99 & 10.5 & 192 & 99 & 98 \\
\hline Furfural & 2 & 86 & 69 & 4 & 88 & 98.5 \\
\hline $\begin{array}{l}\text { Nicotin- } \\
\text { aldehyd }\end{array}$ & 2.5 & 78 & 6.7 & 4.5 & 89 & 14 \\
\hline Crotonaldehyd & 1.5 & 68 & 76 & 3 & 68 & 97 \\
\hline $\begin{array}{l}\text { Phenyl- } \\
\text { acetaldehyd }\end{array}$ & 4 & 82 & 27 & 4.5 & 95 & 40 \\
\hline $\begin{array}{l}\text { 3-Methylthio- } \\
\text { propionaldehyd }\end{array}$ & 3 & 87 & 60 & 6.5 & 97 & 80 \\
\hline Pivalaldehyd & 2.5 & 56 & 45 & 4.5 & 78 & 73 \\
\hline Butyraldehyd & 2 & 75 & 69 & 4.5 & 75 & 96 \\
\hline
\end{tabular}

[a] Als $(R)-(+)$-MTPA-Derivate. 
Der Vergleich zeigt, daß in Essigester zwar längere Reaktionszeiten in Kauf genommen werden müssen, die Enantiomerenreinheit jedoch wesentlich besser ist als bei den Reaktionen in EtOH $/ \mathrm{H}_{2} \mathrm{O}$.

Neuere Untersuchungen, optisch aktive Cyanhydrine mit Dipeptid-Katalysatoren ${ }^{[6]}$ oder durch enzymatische Esterspaltung ${ }^{[7]} O$-acylierter Cyanhydrine herzustellen, verliefen bezüglich der optischen und präparativen Ausbeuten wenig befriedigend.

\section{Arbeitsvorschrift}

A) $\mathrm{Zu} 10 \mathrm{~mL} 0.05$ м Acetat-Puffer (pH 5.4, 50\% Ethanol) werden $150 \mu \mathrm{L}$ Mandelonitril-Lyase-Lösung (700 Einheiten $/ \mathrm{mL}, A_{\mathrm{sp}}=65$ Einheiten $/ \mathrm{mg}$ in $0.02 \mathrm{M}$ Acetat-Puffer, $\mathrm{pH}$ 5.4) pipettier, dann werden $5 \mathrm{mmol}$ Aldehyd 1 und $250 \mu \mathrm{L}(6.5 \mathrm{mmol}) 2$ zugegeben. Man schüttelt bis zur Bildung einer klaren Lösung, lăßt bei Raumtemperatur die in Tabelle 1 angegebene Zeit stehen, extrahiert mit Chloroform, trocknet den Extrakt und entfernt die organische Phase im Rotationsverdampfer.

B) Man lăßt $2 \mathrm{~g}$ Trāger (AViCEL-Cellulose) in $20 \mathrm{~mL} 0.01 \mathrm{M}$ Acetat-Puffer (pH 5.4) 1-2 h quellen. Nach Absaugen, Abpressen und Überführen des Trăgers in einen Einhalskoben lăBt man $150 \mu \mathrm{L}$ Mandelonitril-Lyase-Lơsung (siehe unter A) zutropfen, gibt $20 \mathrm{~mL}$ Ethylacetat (mit $0.01 \mathrm{~m}$ Acetat-Puffer, $\mathrm{pH}$ 5.4, gesăttigt), $5.0 \mathrm{mmol} 1$ und $250 \mu \mathrm{L}(6.5 \mathrm{mmol}) 2 \mathrm{zu}$, la Bt die in Tabelle 1 angegebene Zeit bei Raumtemperatur stehen, saugt und preßt ab, wäscht mit Ethylacetat, trocknet die vereinigten Losungen und entfernt die organische Phase im Rotationsverdampfer.

Beispiel: $0.53 \mathrm{~g}$ Benzaldehyd 1, R=Ph, ergaben nach $\mathrm{A}$ in $1 \mathrm{~h}$ Reaktionszeit $0.66 \mathrm{~g}(99 \%)$ Benzaldehydcyanhydrin $3, \mathrm{R}=\mathrm{Ph},[\alpha]_{0}^{0}=+45^{\circ}\left(c=5, \mathrm{CHCl}_{3}\right)$, $e e=86 \%$. Nach Methode B wurden in $2.5 \mathrm{~h} 0.63 \mathrm{~g}(95 \%) 3, \mathrm{R}=\mathrm{Ph},[\alpha]_{\mathrm{D}}^{20}=$ $+49^{\circ}\left(c=5, \mathrm{CHCl}_{3}\right), e e=99.3 \%$ erhalten.

Eingegangen am 26. Januar, ergănzte Fassung am 16. Februar 1987 [Z 2068]

[1] a) W. Becker, U. Benthin, E. Eschenhof, E. Pfeil, Biochem. Z. 337 (1963) 156; b) W. Becker, E. Pfeil, ibid. 346 (1966) 301; c) W. Becker, H. Freund, E. Pfeil, Angew. Chem. 77 (1965) 1139; Angew. Chem. Int. Ed. Engl. 4 (1965) 1079; E. Pfeil, W. Becker, DBP 1300111 (1969); Chem. Abstr. 72 (1970) P3061t.

[2] E. Hochuli, Helv. Chim. Acta 66 (1983) 489

[3] a) T. Matsuo, T. Nishioka, M. Hirano, Y. Suzukj, K. Tsushima, N. Itaya H. Yoshioka, Pestic. Sci. 1980, 202; b) D. G. Neilson, D. A. V. Peters, L. H. Roach, J. Chem. Soc. 1962, 2272.

[4] a) P. L. Luisi, Angew. Chem. 97 (1985) 449; Angew. Chem. Int. Ed. Engl. 24 (1985) 439; b) A. M. Klibanov, CHEMTECH 1986, 354

[5] a) J. A. Dale, D. L. Dull, H. S. Mosher, J. Org. Chem. 34 (1969) 2534; b) J. D. Elliot, V. M. F. Choi, W. S. Johnson, ibid. 48 (1983) 2294.

[6] a) S. Asada, Y. Kobayashi, S. Inoue, Makromol. Chem. 186 (1985) 1755; b) W. R. Jackson, Brit. Pat. 2143823 (1985), ICI Australian Ltd.; Chem. Abstr. 104 (1986) 68624c.

[7] H. Hirohara, S. Mitsuda, E. Ando, R. Komaki, Stud. Org. Chem. (Amsterdam) 22 (1985) 119; Chem. Abstr. 104 (1986) $67464 \mathrm{p}$.

\section{Decarbonylierungen an Nickel/Kohle-Kontakten* *}

\section{Von Hans Bock* und Olaf Breuer}

\section{Professor Heinz Harnisch zum 60. Geburtstag gewidmet}

Oberflächen-Reaktionen von Gasen an ${ }^{[1 \mathrm{a}-\boldsymbol{c}, \mathrm{f}, \mathrm{g}]}$ und mit $^{\text {[ld.el }}$ Festkörpern lassen sich vorteilhaft durch PE-spektroskopische Echtzeitanalyse ${ }^{[2]}$ des Gasgemisches nach Durchströmen der Reaktionszone auffinden und optimieren (vgl. Abb. 1). Als Modellreaktion für heterogen katalysierte Decarbonylierungen ${ }^{[3 a]}$ haben wir die Spaltung von Crotonaldehyd ${ }^{[3 c]}$ in $\mathrm{CO}$ und Propen bei $10 \mathrm{~Pa}$ an Quarzwolle, an Metallpulvern und an Trägerkontakten unter-

[*] Prof. Dr. H. Bock, Dipl.-Chem. O. Breuer

Institut für Anorganische Chemie der Universität

Niederurseler Hang, D-6000 Frankfurt am Main 50

[**] Oberflächen-Reaktionen, 8. Mitteilung. Diese Arbeit wurde von der Hoechst AG, vom Fonds der Chemischen Industrie und von der MaxBuchner-Stiftung gefördert. - Vorangehende Mitteilungen vgl. [I]. sucht (Schema 1). Bestgeeignet erwies sich ein Nickel/ Kohle-Kontakt ${ }^{[4]}$, bei dem die Decarbonylierungstemperatur um über $500 \mathrm{~K}$ niedriger liegt als bei Quarzwolle ${ }^{[s]}$.

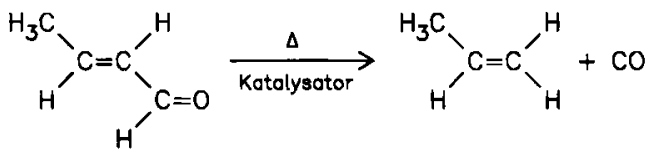

\begin{tabular}{r|llllllll} 
Kotalysator & {$\left[\mathrm{SiO}_{2}\right]$} & {$[\mathrm{Cu}]$} & {$[\mathrm{Ni}]$} & {$\left[\mathrm{Ni} / \mathrm{SiO}_{2}\right]$} & {$[\mathrm{Rh} / \mathrm{C}]$} & $\mathrm{Co} / \mathrm{C}]$ & {$[\mathrm{Ni} / \mathrm{C}]$} \\
\hline$T[\mathrm{~K}]$ & 870 & 770 & 670 & 570 & 520 & 470 & 360
\end{tabular}

Schema 1.

Daß die Reaktion durch die vermutlich in den Poren der oberflächenreichen Kohle vorhandenen Nickelcluster ${ }^{[4]}$ katalysiert wird, zeigt die selektive CO-Abspaltung aus Furfurylaldehyd bei $\mathbf{4 0 0} \mathrm{K}$. Unkatalysiert zerfallt Furfurylaldehyd erst bei $700 \mathrm{~K}$ höherer Temperatur, wobei überwiegend Propin und $\mathrm{CO}$ entstehen $(\mathrm{Abb} .1)^{[3 \mathrm{~b}]}$.

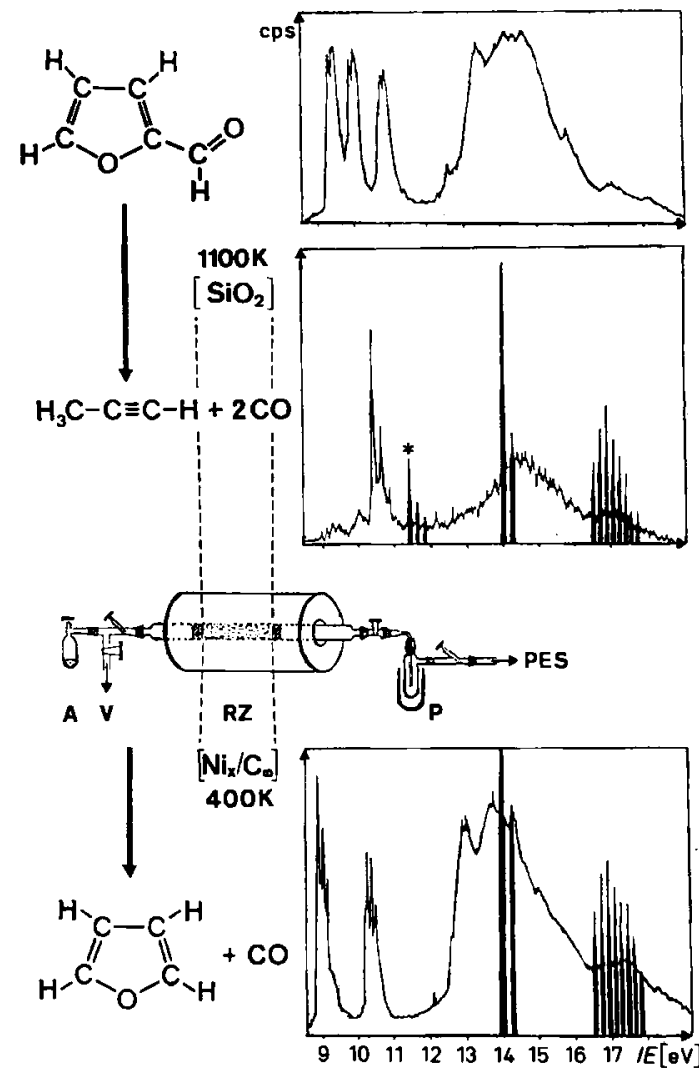

Abb. 1. Thermische Zersetzung von Furfurylaldehyd an Quarzwolle (oben) und selektive Decarbonylierung an einem Nickel/Kohle-Kontakt (unten) He(l)-PE-Spektren und Reaktionsapparatur (A: Ausgangssubstanz, V: Vakuumpumpen-AnschluB, RZ: beheizte Reaktionszone, P; Produkt-Ausfrierfalle für GC-Analyse, PES: Verbindung zum PE-Spektrometer). Als Nebenprodukt der unkatalysierten Zersetzung werden geringe Anteile Acetylen (*) beobachtet. Die schwarzen Banden im mittleren und unteren Spektrum stammen von CO [9]. Das PE-Spektrum von reinem Furan ist bekannt [9]. Die Abwesenheit einer Acetylenbande im unteren Spektrum beweist, daß bei der $\mathrm{Ni}_{x} / \mathrm{C}_{-}$-katalysierten Decarbonylierung von Furfurylaldehyd keine Nebenprodukte entstehen.

Zahlreiche andere organische Carbonylverbindungen lassen sich am Nickel/Kohle-Kontakt ${ }^{[4]}$ bei niedrigen Temperaturen im präparativen Maßstab, und nach Aus- 